\title{
Letter: Bedside Percutaneous Twist Drill Craniostomy of Chronic Subdural Hematoma-A Single-Center Study
}

\author{
Grandhi Aditya ${ }^{1}$ Dimble Raju ${ }^{1} \quad$ Nabanita Ghosh ${ }^{2}$ \\ ${ }^{1}$ Department of Neurosurgery, National Neurosciences Centre, \\ Calcutta, West Bengal, India \\ 2Department of Neuroanesthesiology, National Neurosciences \\ Centre, Calcutta, West Bengal, India
}

J Neurosci Rural Pract:2020;11:506-507

We read the article "Bedside percutaneous twist drill craniostomy of chronic subdural hematoma-a single-center study" by Kidangan et al $^{1}$ with interest. The authors have highlighted their experiences with twist drill craniostomy in chronic subdural hematoma (CSDH) in a series of 80 patients.

The radiological selection criteria on computed tomography (CT) scan, which the authors have adopted for subjecting patients to twist drill craniostomy rather than burr hole evacuation, namely, CSDH with homogeneous hypodensity or isodensity and those with hyperdense gravity-dependent fluid levels, are unexceptionable. However, we feel that even bilateral CSDH with similar radiological features are also good candidates for this procedure, as controlled drainage may allow for uniform brain expansion bilaterally. Although we concur with the authors on other exclusion criteria, Garber et $\mathrm{al}^{2}$ have contended that there are no preoperative imaging characteristics to suggest an advantage of one procedure over the other. Despite the excellent results, the authors report, in their series, that a concern with twist drill craniostomy is inadequate drainage of the subdural hematoma. A recent report by Brazdzionis et $\mathrm{al}^{3}$ advocates the instillation of intracatheter tissue plasminogen activator in patients in whom the repeat CT scan shows unsatisfactory evacuation. It remains to be seen if this technique will be useful in septate CSDH as well and if so, the spectrum of patients treated by this technique might be broadened.

The advantages of a minimally invasive twist drill craniostomy and drainage have been well-described-bedside procedure without monitored anesthesia or anesthesiologist, ${ }^{4}$ lesser analgesic requirements, ${ }^{5}$ smaller incision, ${ }^{4}$ lesser chance of worsening of previous neurodegenerative disorders, ${ }^{5}$ and lesser cost. ${ }^{6}$ Duration of hospital stay also appears to be reduced in patients undergoing a twist drill craniostomy. ${ }^{4,6}$ The authors have inserted an infant feeding tube in the subdural space and connected it with a urobag with gravity-dependent drainage. We are unsure if such a technique would lead
Address for correspondence Prasad Krishnan, MS, MCh, Department of Neurosurgery, National Neurosciences Centre, Peerless Hospital Campus, 2nd Floor, 360, Panchasayar, Garia 700094, Calcutta, West Bengal, India (e-mail: prasad.krishnan@rediffmail.com).

to air entering the subdural space and negate the advantage of a controlled brain expansion, and with our limited experience, we would advocate placing the bag around the patients' "head height."

The site of placement of the twist drill craniostomy (more lateral than a conventional burr hole) and the rationale for the same has been described by the authors. ${ }^{1}$ Others have described the parietal boss ${ }^{3}$ or the area of maximal thickness of the hematoma ${ }^{7}$ as the site of craniostomy. We feel that in addition to concerns about the position of the middle meningeal artery-which the authors have highlighted-a lateral location is safer to prevent injuries to cortical veins that may enter the dura at varying distances from the superior sagittal sinus. Other concerns with twist drill craniostomy include development of extradural hematoma and inadvertent brain penetration. ${ }^{7}$ We avoid these by measuring the thickness of the skull on CT scan and keeping the twist drill bit just a little longer than the same (the base of the drill then acts as the stopper on touching the skin surface), avoiding a blunt drill tip to prevent separation of the dura from the inner table of the skull and not slowing down as the inner table is reached, so that there is a quick clean forceful penetration of the dura.

Finally, this paper is valuable in highlighting the safety and utility of twist drill craniostomy, as several surgeons have a preference for burr hole evacuation (even in radiologically favorable cases of CSDH), based on fear of insufficient anesthesia, reluctance to perform procedure outside the operating room, apprehension of complications, and unwillingness to try a new technique. ${ }^{8}$

\section{Funding}

None.

Conflict of Interest

None declared. 


\section{References}

1 Kidangan GS, Thavara BD, Rajagopalawarrier B. Bedside percutaneous twist drill craniostomy of chronic subdural hematoma-A Single-Center Study. J Neurosci Rural Pract 2020;11(1):84-88

2 Garber S, McCaffrey J, Quigley EP, MacDonald JD. Bedside treatment of chronic subdural hematoma: using radiographic characteristics to revisit the twist drill. J Neurol Surg A Cent Eur Neurosurg 2016;77(3):233-238

3 Brazdzionis J, Patchana T, Wiginton JG IV, Wacker MR, Menoni R, Miulli DE. Intracatheter tissue plasminogen activator for chronic subdural hematomas after failed bedside twist drill craniostomy: a retrospective review. Cureus 2019;11(12):e6472

4 Goyal RK, Nayak B, Maharshi R, Bidhar DD, Panchal S, Pathak HC. Management of chronic subdural hematoma: burr hole versus twist drill - A Prospective Study. Asian J Neurosurg 2018;13(2):319-323

5 Certo F, Maione M, Altieri R, et al. Pros and cons of a minimally invasive percutaneous subdural drainage system for evacuation of chronic subdural hematoma under local anesthesia. Clin Neurol Neurosurg 2019;187:105559

6 Singh SK, Sinha M, Singh VK, et al. A randomized study of twist drill versus burr hole craniostomy for treatment of chronic subdural hematomas in 100 patients. IJNT 2011;08:83-88

7 Yadav YR, Yadav S, Parihar VS. Modified twist drill technique in the management of chronic subdural hematoma. Turk Neurosurg 2013;23(1):50-54

8 Szmuda T, Kierońska S, Słoniewski P, Dzierżanowski J. Modified bedside twist drill craniostomy for evacuation of chronic subdural haematoma. Wideochir Inne Tech Malo Inwazyjne 2019;14(3):442-450 